\title{
LOS DILEMAS DEL PROFESORADO EN LA EDUCACIÓN SUPERIOR MEXICANA
}

El presente trabajo se refiere a las tensiones que los académicos mexicanos experimentan como consecuencia de los desafíos que debe enfrentar la educación superior en México a inicios del siglo XXI. Describe, a grandes rasgos, las condiciones laborales del profesorado mexicano y, especialmente, los problemas inherentes al desempeño de un rol académico cada vez más cuestionado y complejo. Actualmente, se espera que atienda aspectos y realice actividades adicionales a la tríada tradicional relativa al ejercicio de la docencia, la investigación y la difusión.

En primer lugar, se presenta el contexto de la educación superior en el país. Enseguida, se describe la evolución y problemática que enfrenta el académico en México, y se profundiza en la diversidad de funciones y actividades que le son asignadas. En tercer lugar, se reflexiona brevemente sobre el ejercicio de las funciones que realiza para, finalmente, destacar los dilemas en que se ve inmerso en el ejercicio de su rol, al intentar cumplir con la finalidad y funciones que la educación superior actualmente le asigna.

\section{El contexto del trabajo académico en México}

La educación superior en México, así como la de otros países latinoamericanos, se ha caracterizado por orientarse más hacia la actividad de docencia que a la de investigación (Altbach, 1996). A partir de 1970, su crecimiento acelerado y no regulado se vinculó más con la necesidad de atender la creciente demanda estudiantil que con responder a necesidades del desarrollo industrial o tecnológico, así como de la estructura laboral del país. La investigación que se desarrolla en México es escasa y se ha concentrado en las instituciones de educación superior públicas ubicadas principalmente en la Zona 
Metropolitana de la Ciudad de México. Aunque esto tiende a cambiar, la siguiente descripción del sistema de educación superior mexicano se fundamenta en indicadores relacionadas con la docencia.

\section{Una paradoja}

¿Puede un sistema de educación superior crecer de manera notable y, al mismo tiempo, ser más excluyente? Las dinámicas de incremento de la matrícula combinadas con las tendencias demográficas, en un análisis de largo aliento (casi medio siglo), así lo confirman: en 1960 estudiaban el nivel terciario en México menos de 100 mil jóvenes, en medio centenar de instituciones de educación superior (IES), y entonces la tasa bruta de cobertura $(\mathrm{TBC})^{1}$ era del $3 \%$ respecto del grupo de edad respectivo (20-24 años) ${ }^{2}$. Hoy, a mediados de 2008, la matrícula ronda los 2 millones 400 mil estudiantes, la cantidad de instituciones es incomparable (1.774) y la TBC es del 24\% en números redondos, aunque esta varía mucho de acuerdo con el nivel de desarrollo de la región geográfica del país.

La educación superior creció de manera considerable: la TBC se multiplica por un factor de 8; la cantidad de alumnos por $24 \mathrm{y}$ las instituciones son 15 veces más que hace 48 años. Formidable, en un país que, apenas en 1982, pudo estar seguro de contar con un lugar para todos los niños en la escuela básica.

Sin embargo, por la dinámica poblacional, cuando el país tenía una cobertura del 3\%, "dejaba" fuera de la educación superior a casi dos millones de jóvenes; hoy, con todo el crecimiento reportado, se quedan fuera, respecto del grupo etario de 19 a 23 años, ocho millones, dado el cambio en la cantidad de mexicanos por grupo de edad: se pasa de una pirámide clásica, a un romboide. Crecer, sí; márgenes absolutos de exclusión de la oportunidad también creciente, sí: "cosas veredes, Sancho..."

1 La TBC se calcula poniendo en el numerador al total de la matrícula sin control de la edad, sobre el grupo de edad 19-23. Se distingue de la tasa neta de cobertura, en la que se controlan las edades tanto en el numerador como en el denominador.

2 En ese entonces se empleaba el grupo censal 20-24; ahora se usa el de 19 a 23 años. 
Con relación a la cantidad de puestos para el trabajo académico, se pasó de cerca de 10.000 plazas en 1960, a 158.539 en 1999 y a 248.782 en 2005 (OCDE, 2006; ANUIES, 2006). Cerca de dos terceras partes de estas plazas son ocupadas por profesionales contratados por asignatura. Por régimen de financiamiento, se tiene que en 2005 las IES del sector público agrupaban a cerca del $62 \%$ del profesorado (38,5\% de tiempo completo, $7,5 \%$ de tres cuartos y medio tiempo, y $54 \%$ por horas). Mientras que el sector privado concentraba al 38\% restante, pero con una proporción mucho menor de profesores de tiempo completo $(10,2 \%)$ y mucho mayor de profesores contratados por horas (83,9\%) (OCDE, 2006; ANUIES, 2006). En este contexto, descrito muy brevemente, laboran los cerca de 250 mil académicos, cuya caracterización se presenta enseguida.

Un mapa general y desafíos centrales

El sistema de la educación superior con el que cuenta México hoy puede ser resumido atendiendo a ciertas dimensiones generales. 
Tabla 1. Datos básicos del Sistema de Educación Superior Mexicano $2006^{3}$.

\begin{tabular}{|c|c|c|}
\hline \multicolumn{2}{|l|}{ Matrícula escolarizada: } & 2.400 .000 \\
\hline \multicolumn{2}{|c|}{ Tasa Bruta de cobertura, matrícula escolarizada (TBC): } & $24 \%$ \\
\hline Instituciones $^{4}$ & 1.774 & \\
\hline * universitarias & 1.629 & \\
\hline * no universitarias & 127 & \\
\hline * no especificadas & 18 & \\
\hline \multicolumn{3}{|l|}{ Régimen de las instituciones } \\
\hline * públicas & 615 & \\
\hline * privadas & 1.159 & \\
\hline \multicolumn{3}{|l|}{ Matrícula por régimen: } \\
\hline * público & $68 \%$ & \\
\hline * privado & $32 \%$ & \\
\hline \multicolumn{3}{|c|}{ Proporción de la matrícula escolarizada total por nivel } \\
\hline * Técnico superior universitario & Nivel 5B & $3 \%$ \\
\hline${ }^{*}$ Educación normal (licenciatura) ${ }^{5}$ & Nivel 5A & $6 \%$ \\
\hline * Licenciatura universitaria & Nivel 5A & $55 \%$ \\
\hline * Licenciatura tecnológica & Nivel 5A & $30 \%$ \\
\hline * Posgrado & Nivel 6 & $6 \%$ \\
\hline \multicolumn{3}{|c|}{$\begin{array}{l}\text { Distribución social de la escolaridad en México } \\
\text { (Media de años de escolaridad, por quintil de ingreso per cápita) }\end{array}$} \\
\hline *I quintil & 3,8 años & \\
\hline * II quintil & 6,7 años & \\
\hline${ }^{*}$ III quintil & 7,8 años & \\
\hline * IV quintil & 8,4 años & \\
\hline${ }^{*} \mathrm{~V}$ quintil & 12,8 años & \\
\hline
\end{tabular}

Más allá de los sólo aparentemente "fríos" datos, se cuenta con una educación superior que se caracteriza por un enorme crecimiento a lo largo de la segunda mitad del siglo XX e inicios de la presente

3 La información cuantitativa que aquí se muestra, procede del caso mexicano contenido en el "Informe 2007: Educación Superior en Iberoamérica", coordinado por José Joaquín Brunner, editado por CINDA con apoyo de Universia, y que para el caso mexicano tuvo como responsables a Manuel Gil y María Jesús Pérez García.

Puede ser consultado en www.cinda.cl

4 Los datos sobre instituciones son del 2005. Las IES universitarias tienen programas y otorgan grados de licenciatura y posgrado; las no universitarias ofrecen programas de dos años: Técnico Superior Universitario-Profesional Asociado (TSU-PA).

5 La educación normal, dirigida a la formación de profesores en la educación básica, antes era considerada equivalente al bachillerato; ahora se le reconoce como licenciatura, y se separa en las estadísticas educativas en el país.

6 Datos para el año 2000. 
centuria, pero que no alcanza a cubrir ni a una cuarta parte de la población. El nivel de exclusión de esta oportunidad educativa, en términos absolutos, ha crecido, dado el incremento sustancial del grupo etario de referencia.

Es un conjunto de instituciones amplio, en que predominan las universitarias -siguiendo un modelo "clásico o tradicional"-, que no parecen acusar recibo de los cambios en las ofertas institucionales derivadas del cambio de época que ha escenificado el mundo. En las IES del sector público se observa una mayor diversidad de su oferta educativa, así como en las funciones que realizan; las IES privadas, por lo contrario, se orientan sobre todo a la docencia de licenciatura y maestría. A pesar de ser, en términos institucionales, un sistema predominantemente privado, en términos de matrícula solamente un tercio corresponde a dicho sector; entre 1994-1995 y 2004-2005 la contribución del sector privado se incrementó del 20\% al 32\% (ANUIES, 2006), sobre todo como consecuencia de la restricción del cupo en las instituciones públicas, el altísimo costo de las instituciones privadas de elite y, por ende, la apertura de un mercado de instituciones privadas no elitistas que absorben demanda -el Tipo III de la tipología de Levy (1995) - y que carecen de regulación académica profunda por parte del Estado o de alguna agencia pública no estatal.

El sistema sigue concentrando a nueve de cada diez de sus estudiantes en la licenciatura universitaria o tecnológica. A pesar de tener ya 18 años de existencia las, así (mal) llamadas, universidades tecnológicas, diseñadas para absorber al 10\% de la matrícula, apenas cuentan hoy con el 3\%, ello ligado a la ausencia de un proyecto de desarrollo económico que haga necesarios a los técnicos superiores universitarios (TSU) y en un país estancado desde hace al menos dos décadas y en el que existe una cultura de que hay que ser licenciado a toda costa. De ello se sigue la baja proporción del posgrado, más bien atraído por la carrera académica que sigue la consigna de "doctorarse o morir" o al menos tener una maestría. 
Y la desigual distribución social de las oportunidades educativas es palmaria al analizar el promedio de años escolares cursados distinguiendo quintiles de ingreso per cápita, ya que la población que se ubica en el quintil superior cuenta con cerca de 13 años de escolaridad, mientras que la que se encuentra en el inferior tiene poco menos de cuatro.

En este contexto general, la educación superior mexicana enfrenta grandes desafíos. Cuatro de ellos son reconocidos explícitamente, tanto por las mismas instituciones como por las autoridades federales: a) aumentar la cobertura del nivel educativo superior, b) incrementar la pertinencia económica de los programas ofrecidos, c) fortalecer las funciones de investigación y desarrollo tecnológico y, por último, d) hacer más eficiente y transparente la gestión en las IES (ANUIES, 2005). ¿Cómo influyen estos desafíos en la naturaleza de la profesión académica?

\section{El rol del académico en México}

Como fue ejemplificado por Boyer (1990/1997) para el caso estadounidense, las características del rol académico están asociadas con el contexto histórico y socioeconómico en el que funcionan las IES.

A partir de la segunda mitad del siglo pasado, el rol del académico mexicano ha pasado por, al menos, cuatro etapas claramente distinguibles, a pesar de que todavía la última se encuentra en un periodo de definición. En primer lugar y hasta principios de la década de los setenta, puede hablarse del "catedrático", cuyo centro de actividades no estaba ubicado en las IES, sino en una prestigiosa actividad profesional externa a la que le "roba" unas cuantas horas a la semana para impartir alguna clase que le sirve para acrecentar su prestigio. La remuneración que recibe por esta actividad es menor y no se siente comprometido a hacer más de lo que ya hace (Mendieta y Núñez, 1953). 
Con la expansión acelerada de la matrícula a partir de 1970, hubo necesidad de que la enseñanza fuera atendida por personal que se dedicara fundamentalmente a ella. Surge entonces el "docente", quien, a diferencia del catedrático, ya no cuenta en la mayoría de los casos con una práctica profesional externa a su trabajo como profesor de educación superior. Durante esta época crecen los puestos de tiempo completo, antes más bien raros, pero las IES, al verse presionadas para atender a una población estudiantil en aumento permanente, contratan a personal muchas veces sin que hubiese terminado aún sus estudios de licenciatura, al que se tiene que capacitar pedagógicamente (Aguirre Lora, 1988; Gil-Antón y cols., 1994). Años después se formularían diversos programas para fomentar que este mismo personal se profesionalizara disciplinariamente y pudiera asumir, en forma adicional, el rol de investigador.

Con la crisis económica del país, que empieza en 1982, y el dramático descenso de los ingresos del profesorado se hizo evidente que las actividades de investigación (realizadas por una pequeña proporción de académicos de tiempo completo en ciertas IES públicas y en los centros de investigación) corrían el peligro de desaparecer. Ello significaría eventualmente la posible migración de los académicos involucrados hacia los sectores público y privado, y hacia el extranjero inclusive, en búsqueda de mejores condiciones de trabajo y, en particular, de retribución. La creación del Sistema Nacional de Investigadores (SNI) en 1984 -que otorga recursos adicionales por productividad en la investigación- resaltó el papel del académico como "investigador" y justificó que las IES emprendieran programas, muchos de ellos con apoyo federal, para que sus académicos pudieran asumir esta nueva función. A pesar de los muchos esfuerzos institucionales, el nivel de habilitación profesional en investigación de la gran mayoría del personal no permitió que esos programas tuvieran el éxito esperado en el corto plazo, aunque fundaron las bases para que, a principios del siglo XXI, el académico mexicano posea en mayor medida grados más elevados en las disciplinas en las que trabaja y, con ello, esté en condiciones de asumir de manera más exitosa actividades de investigación. 
Si bien hacia finales de los noventa la idea acerca de la dimensión más prestigiosa del rol académico cambió de la docencia a la investigación, a principios de este siglo ha surgido una imagen del académico, al menos en el discurso, más integral y más en sintonía con la características de IES extranjeras y consolidadas, lo cual constituye la cuarta de las etapas planteadas. El programa federal, que se inicia en 1996 y se mantiene con ciertos ajustes hasta el momento, denominado Programa para el Mejoramiento del Profesorado (PROMEP), al hacer depender una serie de apoyos económicos de un "perfil académico deseable", ha promovido la idea de un "académico integral" que desarrolla, al mismo tiempo, actividades de docencia, tutoría de estudiantes, investigación y gestión académica (Urbano Vidales, Aguilar Sahagún y Rubio Oca, 2006).

Las tensiones derivadas de los desafíos que enfrenta la educación superior mexicana

Aunque en cada una de las etapas descritas se han enfatizado ciertas funciones del rol de los académicos, ello no implica que fueran las únicas, sino sólo las principales. No obstante, es claro que la imagen del trabajo académico ha cambiado sustantivamente y se ha hecho más compleja. El cambio, sin embargo, no ha sido independiente de la evolución de la educación superior mexicana y, dado que en estos momentos se encuentra en un proceso de cambio significativo, cabe preguntarse por la forma en que el discurso y la realidad acerca del papel del académico podrían cambiar junto con los desafíos que esta educación pretende enfrentar.

\section{La cobertura de la educación superior}

Mientras que el crecimiento pausado en la cobertura a lo largo de los últimos veinte años generó condiciones para que se reconocieran funciones académicas adicionales a la de docencia, así como para que muchos académicos mejoraran su nivel de formación disciplinaria, un aumento rápido en la matrícula estudiantil atendida puede generar que el rol del académico se retraiga nuevamente hacia la docencia, porque esa es la función clave en un "sistema" de educación superior 
que aumenta rápidamente su cobertura en un contexto de recursos económicos escasos. Asimismo, se abre la posibilidad de que el personal de nuevo ingreso se vuelva a contratar casi en su totalidad, como se hizo durante la primera ola de crecimiento acelerado de los 70, con niveles formativos mínimos de licenciatura, situación que no se ha superado del todo. Otro efecto posible de un incremento drástico en la matrícula es el de solucionar la falta de personal con la contratación de profesores por asignatura que, por su misma condición, tienen pocas posibilidades de involucrarse en la vida institucional más allá de sus actividades directamente relacionadas con sus clases.

Por último, junto con el incremento de la cobertura se requiere que no haya una disminución de la calidad. Para ello se han establecido programas de tutoría de estudiantes que, además, se espera contribuyan a mejorar la eficiencia terminal, también un problema relevante en la educación superior del país. Esta nueva responsabilidad, que se entrega a buena parte del profesorado de la IES, sobre todo a los sujetos al PROMEP, genera una mayor presión sobre las competencias del académico y contrasta con la situación de las IES en otros países, en los cuales existe personal especializado para apoyar el desarrollo de los estudiantes. Como parte de la estrategia para incrementar la cobertura con calidad, al profesorado también se le requiere con mayor frecuencia el uso de nuevas tecnologías de información, complejizando con ello sus tareas.

\section{Pertinencia de la educación superior}

Además de incrementar su cobertura, la educación superior mexicana busca que su oferta educativa sea cada vez más relevante desde el punto de vista económico, y ello ha generado una nueva presión en los académicos. Ya no es suficiente impartir docencia de calidad, sino que ahora se promueve y espera que el académico esté fuertemente vinculado con los sectores productivos de su entorno a través de actividades como asesoría, consultoría o investigación aplicada. Ante la ausencia de condiciones, tanto en términos de tiempo como 
de apoyos, para llevar a cabo adecuadamente estas actividades, los académicos se enfrentan entonces a la disyuntiva de decidir cómo invertir mejor su tiempo entre la docencia y/o investigación, por un lado, y las actividades de vinculación, por otro. Dada la centralidad que la docencia y la investigación tienen en la determinación de la remuneración académica, lo más probable es que la vinculación no relacionada con investigación o desarrollo tecnológico siga ocupando un lugar secundario entre las prioridades reales de los académicos mexicanos.

\section{La investigación y el desarrollo tecnológico}

Mientras que una de las intenciones primeras del SNI fue remediar la disminución en los ingresos de un sector altamente capacitado de los académicos, este programa federal se ha consolidado como uno de los principales promotores de la actividad científica y tecnológica, toda vez que eleva, en periodos multianuales, el ingreso regular de los académicos que forman parte del mismo. No obstante el crecimiento que ha experimentado el SNI en términos de sus miembros, su impacto en la profesión académica en su conjunto es todavía mayor. Más específicamente, al hacer depender un ingreso adicional muy significativo de sus actividades de investigación, los académicos miembros del SNI tienden a enfocar toda su atención en éstas, poniendo en un lejano segundo lugar sus actividades de docencia y gestión, incluyendo en esta última la participación en la vida colegiada de la institución.

Pero como los "académicos SNI" constituyen cerca del 20\% de todos los académicos de tiempo completo, y para las IES es importante mantener ciertos indicadores de calidad que les permitan gestionar mayores presupuestos, entre los que destaca el número de académicos SNI que cada una declara "poseer," ellas pueden resolver su búsqueda simultánea de mayor cobertura y de más investigación y desarrollo tecnológico, separando de muy diversas maneras a los académicos que hacen docencia de los que hacen investigación, reservando para estos últimos las mejores condiciones de trabajo. 
Mejora de la gestión

Por último, la educación superior mexicana busca, desde ya varios años, mejorar y transparentar sus procesos de gestión y administración. En estos esfuerzos, muchas IES han certificado procesos administrativos, reducido personal y asumido políticas administrativas características de organizaciones con fines económicos. Como los académicos parecen participar de una manera limitada en el gobierno de sus instituciones, el componente de gestión en sus actividades tiende a disminuir sobre los hechos, sobre todo en el plano cualitativo, a pesar de que en documentos oficiales se afirme otra cosa.

En la mejora de la gestión se debe considerar que, sobre todo en las IES públicas, los administrativos son los propios académicos que, en su mayoría, no cuentan con la formación para el desempeño de esta labor. En el sector privado, se contratan normalmente administradores de carrera, de manera semejante a como se hace en otros países. Se identifica también una tensión entre los administradores y los académicos; estos últimos manifiestan que cada vez más las decisiones se toman sólo en el plano de lo administrativo sin respetar los procesos del trabajo colegiado; en contraste, los administrativos se quejan del poco interés de aquéllos por participar en comités para la toma de decisiones.

\section{El ejercicio de las funciones académicas}

El foco del trabajo académico en México ha pasado entonces de la actividad docente a un conjunto importante de otras actividades, entre las que destaca la investigación o generación del conocimiento (Boyer, 1990-1997), proceso que se ha vivido más tempranamente en sistemas de educación superior de otros países, como en Estados Unidos. Si se aprende de estas experiencias, se puede apreciar que un énfasis en esta última actividad, con independencia de la diversidad existente entre las IES y académicos, puede conducir a un desequilibrio del trabajo, que se refleja en el sistema de evaluación y promoción de los académicos en el que se privilegia a la investigación por encima de la docencia (Altbach, 1999). Los criterios de evaluación pueden 
influir en la toma de decisiones en cuanto al uso y distribución del tiempo y esfuerzo del profesorado.

La manera en que las diversas funciones del académico pueden combinarse en una determinada IES no puede depender de políticas uniformes y debe estar sujeta a la reflexión. Al analizar la literatura, Braxton (1996) identifica tres diferentes maneras en las que se plantea la relación entre la docencia y la investigación. Primero, la perspectiva del conflicto subraya la diferencia entre estas actividades, lo que requiere de los académicos habilidades y rasgos de personalidad distintos; destaca también la dificultad de desarrollar ambas de manera efectiva y simultánea y señala que la dedicación a la investigación deteriora la calidad y el tiempo que se dedica a la docencia. La perspectiva de complementariedad plantea que tanto la docencia como la investigación contribuyen al logro de la generación, transmisión y aplicación del conocimiento y se refuerzan mutuamente. Una tercera perspectiva sostiene la independencia de ambas actividades, es decir, no compiten entre sí pero tampoco se complementan.

Entre el profesorado mexicano, la elección de ciertas actividades, principalmente orientadas a la investigación, se ha visto influida por los sistemas de estímulos que complementan el salario. Esta forma de diferenciación salarial, se ha generalizado en el sistema de educación superior público a partir de un conjunto de programas federales de incentivos, adicionales a los montos que distribuye el SNI, orientados a reconocer la permanencia, dedicación y el trabajo sobresaliente (Gil Antón, 2004). Aunque las propias IES tienen cierta libertad para orientar y operar sus programas, éstos deben apegarse a la normatividad federal al momento de elaborar sus lineamientos y condiciones. Sin embargo, es común encontrar un mayor énfasis en productos asociados a la investigación en detrimento de las funciones de docencia o difusión (Cordero, Galaz y Sevilla, 2004). Más aún, ha sido tal el peso que han ganado estos programas en la conformación de los ingresos de los profesores que, dependiendo del tipo de IES, los ingresos mediante evaluación periódica pueden constituir más del $50 \%$ de los ingresos contractuales, con el inconveniente que 
no forman parte del conjunto de prestaciones asociadas, entre ellas la jubilación (Gil Antón, 2004). En el caso de las IES privadas, los ingresos mediante programas de incentivos, no superan el 25\% con relación a los salarios contractuales, que en las IES particulares de elite $^{7}$ suelen ser muy atractivos.

Sin duda estos programas han cumplido en parte con su propósito, tanto de mejorar las condiciones salariales del profesorado como de fungir como instrumentos de política de desarrollo en algunas IES. Sin embargo, han generado asimismo una serie de efectos negativos, como la simulación en las actividades que resultan productivas para la obtención de los estímulos y el abandono de aquellas que, aunque importantes, no abonan a un mejor resultado de la evaluación académica.

Más allá de la dicotomía docencia-investigación, es importante que la noción del "académico integral", que se fomenta como parte del PROMEP, promueva que tanto las IES como los académicos y sus grupos (cuerpos académicos y redes temáticas) definan sus prioridades para el trabajo de acuerdo con las diferentes misiones de las IES y los propios intereses de los profesores y grupos, según su disciplina de formación y la etapa de la carrera académica en que se encuentren, entre otros aspectos. En ello los sistemas de evaluación, promoción y estímulo tienen un papel central.

\section{Los dilemas centrales de la profesión académica en México}

El académico mexicano de los últimos treinta años ha sido testigo de una buena cantidad de cambios en el país, en sus IES y, finalmente, en su trabajo como tal. Inmersa en un proceso de consolidación, la profesión académica no ha terminado de construir una ruta

7 Existe la diferenciación entre las IES particulares, en aquellas de prestigio reconocido (ITESM, ITAM, etc.) y las denominadas de absorción de la demanda, las que sustentan su actividad académica predominantemente con profesores por horas. 
de incorporación de nuevos miembros en la que, como en otras profesiones (Young, 1987), sean sus propios miembros los que influyan de una manera decisiva sobre quiénes ingresan, bajo qué condiciones lo hacen y, muy centralmente, cómo avanzan en la jerarquía de puestos y las retribuciones y prestigio asociados.

Si bien se encuentra un mercado de trabajo, las desfavorables condiciones de contratación que se dan en muchos casos (por asignatura y sin posibilidades a corto plazo de obtener estabilidad) planten el dilema de cómo hacer atractiva la carrera académica. Más aún cuando las IES no han desarrollado programas específicos para detectar, reclutar y retener profesionistas talentosos en su planta académica.

El fortalecimiento de esta profesión requiere personal motivado, que se pueda dedicar de tiempo completo y con seguridad laboral a esta profesión, pero respetando la orientación de las IES y la importancia de la vinculación con el campo profesional.

Se requiere de un profesorado que pueda someter a evaluación su trabajo y participar en la toma de decisiones que le compete, en el marco de valores académicos característicos, como la colegialidad o la autonomía, sin caer en una parálisis organizacional o aislamiento que impidan satisfacer adecuadamente los requerimiento de calidad y rendición de cuentas a la sociedad.

La formación del profesorado, a pesar de todos los esfuerzos realizados, está lejos de lograr los niveles que mantienen otros países. En México el 69\% de los académicos es primera generación de su familia en ir a la universidad y el $84 \%$ ha iniciado su carrera académica con sólo la licenciatura (Grediaga, Rodríguez y Padilla, 2004). No obstante, cerca de la mitad del profesorado ha incrementado su escolaridad una vez que inicia la carrera académica, especialmente a partir del PROMEP, aunque con ello se ha logrado mejorar la escolaridad de los profesores de tiempo completo de las IES públicas, a quienes se dirigen los programas. Muchas de las nuevas plazas se 
siguen ocupando por profesores con baja formación, lo que implicará que se sigan destinando recursos para su educación durante su carrera académica.

Las expectativas institucionales actuales, en cuanto a que los académicos lleven a cabo de manera "equilibrada" funciones de docencia, investigación, vinculación y gestión están ejerciendo una presión desmedida sobre ellos y, en muchos casos, obligándolos a realizar sólo las actividades mínimas requeridas en cada uno de los rubros, todo con el fin de cumplir los requisitos que diversos programas demandan para otorgarles remuneraciones adicionales al sueldo ordinario.

\section{Referencias bibliográficas}

Aguirre Lora, G.M.E. (1988) Una crisis dentro de la crisis: La identidad profesional de los docentes universitarios. Revista de la Educación Superior, 27(2), 66, pp. 5-21.

Altbach, Phillip G. G. (ed.) (1996) The international academic profession: Portraits of fourteen countries. Princeton, NJ: Carnegie Foundation for the Advancement of Teaching.

Altbach, Phillip G. (1999). Harsh realities. The professoriate faces a new century. En Altbach, P.G., Berdahl, R. \& Gumport, P. (eds.) American Higher education in the twenty-first century. Baltimore: Johns Hopkins University Press, pp. 271-297.

Asociación Nacional de Universidades e Instituciones de Educación Superior (2006) Consolidación y avance de la educación superior en México: Temas cruciales de la agenda. México: Autor.

Boyer, Ernest L. (1997) Una propuesta para la educación superior del futuro. México: Fondo de Cultura Económica, Universidad Autónoma Metropolitana. (Trabajo original publicado en 1990 bajo el título "Scholarship reconsidered").

Braxton, J.M. (ed) (1996) Faculty teaching and research: Is there a conflict? New Directions for Institutional Research, 90. San Francisco: Jossey-Bass Pub. 
Brunner, José Joaquín (coord.) (2007) Informe 2007: Educación Superior en Iberoamérica, CINDA con apoyo de Universia. (Sitio en Internet) Disponible en www.cinda.cl

Cordero Arroyo, G., Galaz Fontes, J.F., Sevilla García, J.J. (2003) La evaluación de la diversidad en el trabajo académico: Los programas de estímulo de la UABC 1990-2002. México: Asociación Nacional de Universidades e Instituciones de Educación Superior y Universidad Autónoma de Baja California.

Gil Anton, Manuel y cols. (1994). Los rasgos de la diversidad: Un estudio sobre los académicos mexicanos. México: Universidad Autónoma Metropolitana, Unidad Azcapotzalco.

Gil Antón, Manuel (2005) El crecimiento de la educación superior privada en México: de lo pretendido a lo paradójico: ¿o inesperado? Revista de la Educación Superior. Vol. XXXIV (1), 133, pp. 9-20.

Gil Antón, Manuel (2004) Amor de ciudad grande: Una visión general del espacio para el trabajo académico en México. En Altbach, P. (coord.) El ocaso del gurú. La profesión académica en el tercer mundo. México: UAM, pp. 43-81.

Grediaga, Rocío, Rodríguez, Raúl y Padilla, Laura (2004) Políticas públicas y cambios en la profesión académica en México en la última década. México: ANUIES.

Levy, Daniel C. (1995) La educación superior y el Estado en Latinoamérica. Desafios privados al predominio público. México: CESU-FLACSO-Porrúa.

Mendieta y Núñez, L. (1953) Ensayo sociológico sobre la universidad. En Mendieta y Núñez L. (coord.) Primer censo nacional universitario, 1949 México: Universidad Nacional Autónoma de México, pp. XIX-CIV.

OCDE (2006) Análisis Temático de la Educación Terciaria. México. Nota de país.

Urbano Vidales, Guillermina, Aguilar Sahagún, Guillermo y Rubio Oca, Julio (2006) Programa de Mejoramiento del Profesorado: Un primer análisis de su operación e impactos en el proceso de fortalecimiento académico de las universidades públicas. México: Secretaría de Educación Pública.

Young, R.E. (1987). Faculty development and the concept of "profession". Academe, 73(3), pp. 12-14.

Recibido: 15 de mayo de 2008

Aceptado: 3 de junio de 2008 